\title{
The future of social media in orthopedic surgery
}

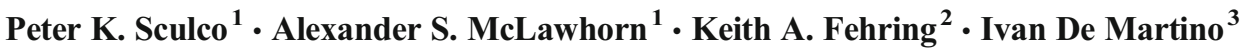

Published online: 29 April 2017

(C) Springer Science+Business Media New York 2017

In $2015,84 \%$ of adults used the Internet on a regular basis and $80 \%$ of these user references online sources for health-related information $[1,2]$. There has been a corresponding growth in online social networking and social media outlets, in the form of websites, online social networks, and mobile applications [3]. These social media platforms have expanded to become vital networks for patients and physicians to communicate with each other, among each other, and learn from each other's experiences. With an estimated 6.5 million health-related search engine queries per day [4], we believe that the contemporary practicing physician must be aware of these social media outlets and ideally have a deeper understanding and utilization that will allow for the improvement of patient care, patient and physician education, and increases in practice outreach, scope, and volume.

As previously discussed, social media grew with the development of Web 2.0 which allowed for a more dynamic interaction between the user and the web. Web 2.0 allowed for the Internet user to create content in the form of personal blogs, to post messages in online discussion boards, and to create

This article is part of the Topical Collection on Social Media and Orthopedics

Peter K. Sculco

sculcop@hss.edu

1 Adult Reconstruction and Joint Replacement Division, Department of Orthopaedic Surgery, Hospital for Special Surgery, 535 East 70th Street, New York, NY 10021, USA

2 Hip and Knee Center, OrthoCarolina, 2001 Vail Avenue Suite 200A, Charlotte, NC 28207, USA

3 Complex Joint Reconstruction Center, Department of Orthopaedic Surgery, Hospital for Special Surgery, 535 East 70th Street, New York, NY 10021, USA online profiles, and eventually online social networks. Going forward, practicing orthopedic surgeons, physician groups, and hospital networks have a decision, either control social media outlets or be controlled by them. Current social media platforms such as Facebook, Twitter, YouTube, and Instagram offer far-reaching online social networks that will grow in usage over the next few years. These social media outlets will continue to be a platform for physicians to improve practice visibility with each application potentially serving its own function. Facebook will provide an online social network for the practice. YouTube will provide preoperative, perioperative, and postoperative video instructions. Instagram will serve as a way to share patient photos and progress and provide helpful information during the recovery process.

The benefit of increasing physician-specific online content is that patient education and the surgeon-patient engagement can potentially occur remotely. Social media will evolve into a form of telemedicine where patients will be guided through the preoperative, perioperative, and postoperative period with a digital assistant or orthopedic avatar. Patients will be able to post their results on a physician-controlled or hospitalcontrolled health care network. Radiographic and clinical performance can then be tracked remotely and patients will have the choice to follow up in the physician office or remotely from home. It is likely than in the next 5 years, telemedicine and remote follow-up will be a common practice for standard follow-up of the orthopedic patient. In addition, the additional online content in the form of patient education forms, online videos, physical therapy exercises, and motivation milestones will allow the patient to have more digital contact with the physician office without an increase in overall face-to-face time. Increased patient engagement without an increase in physician involvement allows for potentially more costeffective care delivery. In the era of bundles payments, any technology that continues to provide a high level of patient 
care at a reduced cost will succeed. Social media telemedicine platforms fulfill the value-proposition and will likely be embraced by physicians and hospital networks in the near future.

In addition, standardized electronic medical record (EMR) will ultimately provide a comprehensive online fully integrated platform for physician-patient engagement and interaction. Not only the patients will be able to see all of their clinical information but they will also be able to contact the physician or other health care providers with specific questions and requests. This will allow for a patient centered dialogue that will be automatically recorded in the medical record.

Finally, social media tools are already becoming an extension of the exam room, board room, operating room, and OR lounge. For the physician-to-physician relationship, social media will continue to become increasingly interactive. Currently, online webinars allow for immediate interaction between health care practitioners allowing them to ask and receive answers immediately. The next step will likely be into the operating room where surgeons from anywhere in the world will be able to receive real-time feedback and advice during challenging cases.

A first attempt of combining the real-time augmented reality and wearable computing devices such as Google Glass (Google Inc., Mountain View, CA, USA) was made few years ago [5]. During a total shoulder replacement, the local surgeon was able to interact with the remote surgeon within the local surgical field. New technological offerings such as HoloLens (Microsoft Corporation, Redmond, WA, USA) allow for a more advanced augmented reality with the use of holographic images that will be viewable to both the user and a remote user who will be able to visualize the same augmented reality. This new technology platform may allow surgeons to interact in real-time and share an augmented reality remotely. This type of surgeon-to-surgeon educational platform could allow for added integration and collaboration that will further enhance surgeon-to-surgeon education.

There are several risks and limitations associated with the increasing usage of social media in orthopedic surgery. As physicians develop a larger social media presence, management of these accounts will become increasingly complicated. Already, several companies offer services to manage medical social media accounts. While this may reduce the time required to manage these online platforms, the physician is ultimately responsible and must control content production in order to prevent independent parties with minimal medical training from producing unreliable content. As social media becomes increasingly mainstream, finding the correct balance between maintaining a time-efficient strategy while controlling the content quality will be difficult to balance. In addition, all of these endeavors have potential patient privacy concerns and all the medical-legal issues that apply to any situation where patient data is shared and clinical care is delivered. Patient privacy and safety are paramount in the unveiling of any new technology, and social media platforms must be held to the same high standards similar to the release of a new medical product.

In summary, the future of social media is bright. For even the most basic users, the use of online platforms to gain access to new patients and offer online educational content will become commonplace. For the more interested practitioners who are interested in managing multiple platforms, each user must determine the level of commitment and comfort in outsourcing portion on ones online image to third party organizations. From a care delivery standpoint, social media will improve patient engagement without increasing cost. Social media will further enable remote follow-up visits which will save time and cut cost for both the surgeon and patient alike. And finally, surgeon education will move from Web 2.0 to Web 3.0 where the real-time interaction will occur not just at desktop computer or mobile device, but in the operating room when immediate assistance is needed to help in a complex case. Social media is currently changing the way we deliver almost every aspect of patient care. Understanding the risks and benefits of this powerful technology is essential for continued success in the current medical environment.

\section{Compliance with ethical standards}

Conflict of interest All authors declare that they have no conflict of interest.

Human and animal rights and informed consent This article does not contain any studies with human or animal subjects performed by any of the authors.

\section{References}

1. Perrin A, Duggan M. Americans' Internet Access: 2000-2015. Pew Internet \& American Life Project. Washington, DC: Pew Research Center. 2015. http://www.pewinternet.org/2015/06/26/americansinternet-access-2000-2015/. Accessed 16 Aug 2016.

2. Fox S. The Social Life of Health Information, 2011. Pew Internet \& American Life Project. Washington, DC: Pew Research Center. http://pewinternet.org/Reports/2011/Social-Life-of-Health-Info. aspx. Accessed 16 Aug 2016.

3. Perrin A. "Social Networking Usage: 2005-2015." Pew Research Center. 2015. Available at: http://www.pewinternet.org/2015/10/08/ 2015/Social-Networking-Usage-2005-2015/. Access 16 Aug 2016.

4. Obar JA, Wildman S. Social media definition and the governance challenge: an introduction to the special issue. Telecommun Policy. 2015;39(9):745-50. doi:10.1016/j.telpol.2015.07.014.

5. Ponce BA, Menendez ME, Oladeji LO, Fryberger CT, Dantuluri PK. Emerging technology in surgical education: combining real-time augmented reality and wearable computing devices. Orthopedics. 2014;37(11):751-7. doi:10.3928/01477447-20141023-05. 